\title{
Efeitos do treino com plataforma vibratória sobre a força muscular em idosas hígidas
}

\author{
Bárbara da C. Flores, ${ }^{1}$ Greice Raquel Machado, ${ }^{2}$ Kauan P. Pinto,${ }^{3}$ Nadiéle C. Fischer, ${ }^{4}$ Caroline Franciele \\ Waechter, ${ }^{3}$ Dannuey M. Cardoso, ${ }^{5}$ Litiele Evelin Wagner, ${ }^{6}$ Isabella M. de Albuquerque, ${ }^{7}$ Mario Bernardo- \\ Filho, ${ }^{8}$ Dulciane N. Paiva ${ }^{9 *}$
}

\begin{abstract}
Resumo
Introdução: A vibração de corpo inteiro (VCI) empregue através da plataforma vibratória (PV) tem sido utilizada como método coadjunto à reabilitação física, ocasionando redução da perda mineral óssea e aumento do desempenho físico em idosos. Objetivos: O presente estudo objetivou avaliar os efeitos da VCI por meio da PV sobre a força muscular de membros inferiores de idosas hígidas. Métodos: Estudo experimental que avaliou idosas hígidas alocadas randomicamente no Grupo Plataforma Vibratória (GPV), que realizaram treino na PV; e no Grupo Controle (GC), no qual as idosas permaneceram sobre a PV desligada durante 20 minutos. Avaliou-se o perfil antropométrico e a força muscular do membro inferior por meio do Teste de 1 Repetição Máxima (1-RM) antes e após dois meses de treinamento com VCI através da PV com frequência de $35 \mathrm{~Hz}$ e amplitude de $2 \mathrm{~mm}$, durante 2 meses, três vezes na semana com 1 minuto de treino intercalado com 1 minuto de descanso, totalizando 20 minutos de treinamento em posição ortostática. Resultados: Amostra (n=17, GPV: $n=7$ e GC: $n=10$ ) com média de idade de 65,4 46,6 anos. Houve aumento significativo da força muscular dos adutores de quadril $(p=0,014)$ e dos músculos extensores de joelho $(\mathrm{p}=0,012)$. Conclusões: A VCI ocasionou aumento da força muscular de membros inferiores das idosas hígidas avaliadas.
\end{abstract}

Descritores: Envelhecimento; Força muscular; Terapias complementares.

\section{Abstract \\ Effects of vibrating platform training on muscle strength in healthy elderly women}

Introduction: Whole body vibration (WBV) used through a vibratory platform (VP) has been used in conjunction with physical rehabilitation, reducing bone mineral loss and increasing physical performance in the elderly. Objectives: The present study aimed to evaluate the effects of WBV through VP on lower limbs muscle strength of healthy elderly women. Methods: An experimental study that evaluated healthy elderly women randomly allocated, who underwent training in the Vibration Platform (VP), and in the Control Group (CG), in which the elderly women remained on the VP switched off for 20 minutes. The anthropometric profile and muscle strength of the lower limb were evaluated through the 1-repetition maximal test (1-RM) before and after two months of WBV training on the VP with frequency of $35 \mathrm{~Hz}$ and amplitude of $2 \mathrm{~mm}$ for 2 months, three times a week with 1 minute of training alternating with 1 minute of rest, totaling 20 minutes
1. Programa de Residência Multiprofissional em Saúde. Hospital Santa Cruz. Santa Cruz do Sul, RS, Brasil.

2 Departamento de Biologia e Farmácia. Curso Superior de Tecnologia em Estética e Cosmética. Universidade de Santa Cruz do Sul (UNISC). Santa Cruz do Sul, RS, Brasil.

3 Programa de Iniciação Científica. Universidade de Santa Cruz do Sul (UNISC). Santa Cruz do Sul, RS, Brasil.

4 Programa de Iniciação Científica (PUIC). Universidade de Santa Cruz do Sul (UNISC). Santa Cruz do Sul, RS, Brasil.

5 Programa de Pós-Graduação em Ciências Pneumológicas. Universidade Federal do Rio Grande do Sul (UFRGS). Porto Alegre, Brasil.

6 Programa de Iniciação Científica (FAPERGS). Universidade de Santa Cruz do Sul (UNISC). Santa Cruz do Sul, RS, Brasil.

7 Programa de Pós-Graduação em Reabilitação Funcional. Departamento de Fisioterapia e Reabilitação. Universidade Federal de Santa Maria (UFSM). Santa Maria, Brasil.

8 Departamento de Biofísica e Biometria. Universidade do Estado do Rio de Janeiro. Rio de Janeiro, RJ, Brasil. Instituto Nacional do Câncer. Rio de Janeiro, RJ, Brasil.

9 Programa de Pós-Graduação em Promoção da Saúde. Departamento de Educação Física e Saúde. Universidade da Santa Cruz do Sul (UNISC). Santa Cruz do Sul, RS, Brasil.

*Endereço para correspondência:

Avenida Independência, 2.293

Santa Cruz do Sul, RS, Brasil. CEP: 96815-900.

E-mail:dulciane@unisc.br

Revista HUPE, Rio de Janeiro, 2018;17(1):17-21

doi: 10.12957/rhupe.2018.39271

Recebido em 12/12/2017. Aprovado em 27/03/2018.

of training in the orthostatic position. Results: Sample ( $n=17$, VPG: $\mathrm{n}=7$ and GC: $\mathrm{n}=10$ ) with mean age of $65.4 \pm 6.6$ years. There was a significant increase in the muscle strength of the hip adductors $(\mathrm{p}=0.014)$ and the knee extensor muscles ( $\mathrm{p}=0.012$ ). Conclusions: The WBV caused an increase in lower limbs muscle strength of the analyzed healthy elderly women.

Keywords: Aging; Muscle strength; Complementary therapies.

\section{Resumen}

Efectos da Plataforma vibratoria sobre la fuerza muscular de las personas mayores 
Introducción: La vibración de cuerpo entero (VCI) empleada a través de la plataforma vibratoria (PV) ha sido utilizada como método coadjunto a la rehabilitación física, ocasionando reducción de la pérdida mineral ósea y aumento del desempeño físico en ancianos. De esta forma, el presente estudio objetivó evaluar los efectos de la VCI por medio de la PV sobre la fuerza muscular de miembros inferiores de ancianas hígidas. Métodos: Estudio experimental que evaluó ancianas hígidas asignadas al azar en el Grupo Plataforma Vibratoria (GPV), que realizaron entrenamiento en la Plataforma Vibratoria (VP) y en el Grupo Control (GC), en el cual las ancianas permanecieron sobre la PV apagada durante 20 minutos. Se evaluó el perfil antropométrico y la fuerza muscular del miembro inferior a través del Test de 1 Repetición Máxima (1-RM) antes

\section{Introdução}

O envelhecimento é caracterizado por alterações biopsicossociais específicas que aumentam a vulnerabilidade e os problemas relacionados à saúde, como a atrofia muscular e o comprometimento funcional. Tais aspectos afetam significativamente o estado geral de saúde dos idosos, aumentando a morbidade desta população., ${ }^{1,2}$

Estima-se que em 2050 o número de idosos estadunidenses aumente para 80 milhões, correspondendo a $20 \%$ da população dos Estados Unidos. No Brasil, estima-se que em 2030 haverá cerca de 40,7 milhões de pessoas com idade acima de 65 anos, sendo a expectativa de vida para esse ano de 77,4 anos, ${ }^{3}$ o que torna necessária a adoção de medidas que promovam maior independência e reduzam a fragilidade deste grupo demográfico, sendo este um dos principais desafios enfrentados pelo sistema de saúde. ${ }^{4}$

A fraqueza muscular está fortemente associada à redução da mobilidade e ao aumento do risco de quedas e de deficit de equilíbrio em idosos. ${ }^{5}$ Portanto, a avaliação da força muscular é fundamental dentro da anamnese geriátrica. Nesse sentido, o método de 1 Repetição Máxima (1-RM) pode ser usado para avaliar o deslocamento da maior carga possível para um determinado tipo de exercício. ${ }^{6}$

Dentre os inúmeros meios para reduzir os efeitos deletérios do envelhecimento, a vibração de corpo inteiro (VCI) empregue através da plataforma vibratória (PV) tem sido utilizada com resultados particularmente satisfatórios na redução da perda mineral óssea ${ }^{7}$ e aumento da força muscular, da potência e do desempenho físico em idosos, ${ }^{8}$ podendo ainda ser um método coadjunto ao tratamento fisioterapêutico e no treinamento esportivo. ${ }^{9} \mathrm{~A}$ VCI apresenta potencial para y después de dos meses de entrenamiento con VCI a través de la PV con frecuencia de $35 \mathrm{~Hz}$ y amplitud de $2 \mathrm{~mm}$, durante 2 meses, tres veces en la semana con 1 minuto de entrenamiento intercalado con 1 minuto de descanso, totalizando 20 minutos de entrenamiento en posición ortostática. Resultados: Muestra ( $\mathrm{n}=17$, GPV: $\mathrm{n}=7$ y GC: $\mathrm{n}=10$ ) con una media de edad de 65,4 $\pm 6,6$ años. Se observó un aumento significativo de la fuerza muscular de los aductores de cadera $(\mathrm{p}=0,014)$ y de los músculos extensores de rodilla ( $\mathrm{p}=0,012$ ). Conclusión: A VCI ocasionou aumento da força muscular de membros inferiores das idosas hígidas avaliadas..

Palabras clave: Envejecimiento; Fuerza muscular; Terapias complementarias.

atuar como coadjuvante na prevenção e tratamento de osteoporose em mulheres em pós-menopausa, ${ }^{10}$ na melhora do controle glicêmico em portadores de diabetes mellitus tipo $2,{ }^{11}$ na redução dos sintomas da síndrome das pernas inquietas ${ }^{12}$ e na melhora da incontinência urinaria de esforço, ${ }^{13}$ proporcionando também redução do tônus muscular patológico em crianças com paralisia cerebral. ${ }^{14}$

Segundo Zhang e colaboradores, ${ }^{15}$ o treino com VCI resulta em aumento da mobilidade, do equilíbrio dinâmico e do estado geral de saúde de idosas hígidas. Tais resultados foram corroborados por metanálise que evidenciou que a $\mathrm{VCI}$ pode ser utilizada em detrimento dos exercícios convencionais na população idosa. Entretanto, há escassez de relatos sobre os efeitos da VCI na capacidade funcional de idosos hígidos, ${ }^{16} \mathrm{o}$ que motivou o presente estudo a avaliar o efeito do treino de vibração de corpo inteiro sobre a força muscular de membros inferiores de idosas hígidas.

\section{Materiais e métodos}

Trata-se de estudo experimental que submeteu idosas com faixa etária de 60 a 81 anos do munícipio de Santa Cruz do Sul, RS, ao treino com PV. O acesso à amostra ocorreu por chamada pública por meio de e-mail institucional da Universidade de Santa Cruz do Sul, Santa Cruz do Sul, RS. O estudo atendeu aos critérios da resolução vigente do Conselho Nacional de Saúde sobre ética em pesquisa com seres humanos (466/12), sendo aprovado pelo Comitê de Ética em Pesquisa em Seres Humanos da Universidade de Santa Cruz do Sul (UNISC), sob parecer nº 1.378.420. Após fornecer informações detalhadas sobre a pesquisa e os procedimentos aos quais as idosas foram 
submetidas, obteve-se a assinatura do Termo de Consentimento Livre e Esclarecido. Ressalta-se que o presente estudo consiste em braço temático do estudo intitulado "Efeitos da Plataforma Vibratória sobre a composição corporal, força muscular, flexibilidade e perfil bioquímico de idosas hígidas", aprovado pelo CEP UNISC sob o número de protocolo 1.300.686.

A amostra foi alocada randomicamente no Grupo Controle (GC) e no Grupo Plataforma Vibratória (GPV) por meio de sorteio determinado por envelope opaco e lacrado. Foram incluídas mulheres insuficientemente ativas de acordo com os critérios do International Physical Activity Questionnaire (IPAQ), ${ }^{17}$ com massa corporal máxima de $159 \mathrm{~kg}$ e índice de massa corporal (IMC) entre 18 e 39. Foram excluídas aquelas com hipertensão arterial sistêmica sem controle medicamentoso, com neuropatia que comprometesse a cognição, idosas submetidas a procedimentos cirúrgicos, com colocação de prótese óssea ou marcapasso cardíaco, labirintite, hérnia de disco, trombose, diabetes descompensada ou câncer, deficit cognitivo ou ortopédico que impossibilitasse a participação no estudo ou que faltassem a três sessões consecutivas ao treino na plataforma vibratória.

Foi realizada a avaliação antropométrica, sendo a estatura (m) aferida por meio de estadiômetro (Profissional Sanny ${ }^{\circledR}$, Brasil), a massa corporal $(\mathrm{kg})$ foi aferida por meio de balança digital (Modelo BAL-150 PA, Brasil) e o IMC, por meio da razão entre a massa corporal e a estatura elevada ao quadrado. Antes do início de cada sessão de treinamento na PV foi realizada anamnese e exame físico para avaliação dos sinais vitais. A pressão arterial sistólica(PAS) e a pressão arterial diastólica (PAD) foram avaliadas por meio do método auscultatório, a frequência cardíaca (FC) e da saturação periférica de oxigênio (SpO2) foram avaliadas por oximetria de pulso (Oxy Control Geratherm ${ }^{\circledR}$ - Alemanha) e a frequência respiratória (FR) pela contagem do número de incursões respiratórias por minuto (irpm).

\section{Treinamento na plataforma vibratória}

A amostra foi submetida ao treino na PV (Power Plate ${ }^{\circledR}$, modelo $m y 7^{\mathrm{TM}}$, Reino Unido) com frequência de $35 \mathrm{~Hz}$ e amplitude de $2 \mathrm{~mm}$, durante 2 meses, três vezes na semana com 1 minuto de treino intercalado com 1 minuto de descanso, totalizando tempo total de treinamento de 20 minutos na posição ortostática. ${ }^{18-20}$ Ressalta-se que os indivíduos alocados no GC permaneceram sobre a PV desligada durante 20 minutos.

\section{Teste de Uma Repetição Máxima}

A realização do teste de uma repetição máxima (1-RM0) foi iniciada bilateralmente e com a imposição gradativa de sobrecarga, em que as voluntárias realizaram uma repetição com o máximo de peso possível. ${ }^{21} \mathrm{O}$ referido teste foi aplicado no leg press inclinado a $45^{\circ}$, onde a participante realizou extensão do joelho com o quadril flexionado, tendo seu início em sedestação com a coluna apoiada no encosto e pés posicionados sobre a plataforma do aparelho. $\mathrm{Na}$ fase excêntrica do movimento foi realizada extensão de joelhos e na fase concêntrica foi realizada a flexão de joelhos a $90^{\circ} .22$ Após, foi implementada a adução de quadril contra resistida com a participante em sedestação na cadeira adutora, com quadril e joelhos posicionados a $90^{\circ}$. Adotou-se intervalo de 3 a 5 minutos para acréscimo de peso a cada repetição e, diante da impossibilidade da execução de uma repetição, uma segunda tentativa do teste foi realizada. ${ }^{23}$

\section{Análise estatística}

A análise dos dados foi realizada com o programa Statistical Package for Social Science (versão 23.0, EUA), sendo a normalidade avaliada por meio do teste de Shapiro Wilk. Dados apresentados em média e desvio padrão. Para comparar os grupos quanto às características antropométricas e força muscular foi utilizado o Teste $t$ Student. Para efeito de significância estatística foi adotado um $\mathrm{p}<0,05$.

\section{Resultados}

A amostra foi composta por 17 idosas com média de idade de $65,4 \pm 6,6$ anos e IMC de $28,2 \pm 3 \mathrm{~kg} / \mathrm{m}^{2}$ (GC: $\mathrm{n}=10$; e GPV: $\mathrm{n}=7$ ). As características das amostras avaliadas foram homogêneas entre os grupos e podem ser observadas na Tabela 1.

Em relação à força muscular aferida pelo Teste

Tabela 1. Caracterização da amostra avaliada

\begin{tabular}{lcccc}
\hline \multicolumn{1}{c}{ Variáveis } & $\mathbf{G C}(\mathbf{n}=\mathbf{1 0}) *$ & $\mathbf{G P V}(\mathbf{n}=\mathbf{7})^{*}$ & $\mathbf{P}$ \\
\cline { 1 - 2 } \cline { 5 - 5 } Idade (anos) & $65,4 \pm 6,6$ & $66,8 \pm 4,3$ & 0,385 \\
\hline Massa corporal $(\mathrm{kg})$ & $69,8 \pm 8,0$ & $70,6 \pm 10,1$ & 0,963 \\
\hline Estatura $(\mathrm{cm})$ & $1,59 \pm 0,05$ & $1,60 \pm 0,07$ & 0,977 \\
\hline $\mathrm{IMC}\left(\mathrm{kg} / \mathrm{m}^{2}\right)$ & $27,6 \pm 2,7$ & $27,5 \pm 2,9$ & 0,590 \\
\hline
\end{tabular}

Legenda: GC: grupo controle; GPV: grupo plataforma vibratória; IMC: índice de massa corporal. *Valores em média e desvio padrão $(p<0,05)$.. 


\section{Artigo original}

de 1-RM, não foi detectada diferença entre os grupos analisados. Constatou-se aumento da força muscular dos adutores de quadril no GPV $(p=0,014)$, que apresentou valores de $66,1 \pm 22,8 \mathrm{~kg}$ antes da intervenção e de $72 \pm 20,3 \mathrm{~kg}$ após o período de treinamento na $\mathrm{PV}$, bem como da cadeira extensora de joelho no desenvolvimento do leg press do pré $(46,4 \pm 19,7 \mathrm{~kg})$ para o pós treinamento na PV $(75,0 \pm 16,5 \mathrm{~kg})(\mathrm{p}=0,012)$.

\section{Discussão}

O presente estudo avaliou a força muscular de idosas hígidas antes e após o período de oito semanas de treinamento com plataforma vibratória, tendo evidenciado aumento significativo na força muscular de adutores de quadril e extensores de joelho antes e após período de treinamento na PV.

O envelhecimento aumenta a susceptibilidade para o desenvolvimento de incapacidades e enfermidades crônicas, estando esta população especialmente vulnerável ao adoecimento e às mudanças morfológicas. ${ }^{24}$ A redução da força muscular e da capacidade funcional se torna evidente, determinando a perda progressiva de adaptação de idosos ao meio ambiente. ${ }^{25}$

A VCI tem sido estudada como um recurso alternativo para realização de exercícios e aumento da capacidade física, ${ }^{26}$ tendo o presente estudo evidenciado que o treino na PV aumentou a força muscular dos adutores de quadril e dos extensores de joelho. A inatividade física está entre as principais causas de redução da força muscular entre os idosos e, diante disso, o treino na PV torna-se uma medida alternativa para a indução de melhorias na força muscular desta população. ${ }^{27}$

Ferreira e colaboradores ${ }^{28}$ implementaram o treino com PV com frequência de $30 \mathrm{~Hz}$ (4-6 mm), por 90 segundos, 1 vez por semana com o indivíduo em posição de semiflexão de joelhos e evidenciaram que o estímulo vibratório não apresentou efeito agudo sobre a potência muscular de adultos ativos. Com o envelhecimento, a força muscular extensora de joelho de idosos apresenta maior redução se comparada aos extensores de cotovelo, ${ }^{29}$ devendo ser ressaltado que a perda de massa muscular ocorre predominantemente em membros inferiores, devido à redução da atividade física relacionada com o avançar da idade. ${ }^{30}$ Segundo Urso e colaboradores, ${ }^{31}$ o treinamento de força em idosos com e sem presença de VCI implementado com frequência de $30 \mathrm{~Hz}$ (2-4 mm), 2 vezes por semana durante 10 semanas com o indivíduo em semiflexão de joelhos proporciona desempenho semelhante no teste de 1-RM entre os grupos intervenção e controle. Em

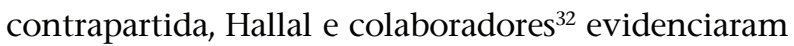
aumento da força e da potência dos músculos dos membros inferiores de adultas jovens submetidos ao treino com haste vibratória com frequência de $5 \mathrm{~Hz}$ por 50 minutos, 2 vezes por semana, durante 8 semanas.

A VCI pode ser utilizada como modalidade de treinamento complementar para idosos hígidos e para indivíduos com lesões musculoesqueléticas,, ${ }^{33-35}$ visto que há evidências de que o aumento da força muscular de membros inferiores resulta em aumento no equilíbrio dinâmico de idosos. ${ }^{36} \mathrm{O}$ estímulo dos fusos musculares e dos neurônios motores alfas pela VCI ocasiona contração muscular ${ }^{37}$ e aumenta a efetividade da atividade muscular durante treinamento de longo prazo. $^{38}$

A resposta neuromuscular à VCI depende do tipo, frequência, amplitude e duração do estímulo vibratório, bem como da posição que o indivíduo adota sobre a PV..$^{39}$ Nesse sentido, a VCI é uma modalidade terapêutica que ocasiona aumento da força muscular esquelética e da qualidade de vida do idoso. ${ }^{40}$

Deve ser ressaltado que o presente estudo apresentou algumas limitações como o fato de não ter sido desenvolvido um grupo placebo, entretanto, advindo dos resultados parciais obtidos, conclui-se que a PV ocasionou aumento da força muscular dos membros inferiores das idosas hígidas avaliadas. A continuidade do estudo torna-se importante para que se comprove a segurança do método, visto que os possíveis efeitos deletérios causados pela VCI na população idosa ainda não estão completamente elucidados.

\section{Referências}

1. Abrahin O, Rodrigues RP, Nascimento VC, et al. Single-and multiple-set resistance training improves skeletal and respiratory muscle strength in elderly women. Clin Interv Aging. 2014;9(1):1775-1782.

2. Amaral JF, Alvim FC, Castro EA, et al. Influence of aging on isometric muscle strength, fat-free mass and electromyographic signal power of the upper and lower limbs in women. Braz J Phys Ther. 2014;18(2):183-190.

3. Silva IA, Amorim JR, Carvalho FT, et al. Efeito de um protocolo de Facilitação Neuromuscular Proprioceptiva (FNP) no equilíbrio postural de idosas. Fisioter Pesqui. 2017;24(1):62-67.

4. Ali S, Garcia JM. Sarcopenia, cachexia and aging: diagnosis, mechanisms and therapeutic options. Gerontol. 2014;60(4):294-305.

5. Borde R, Hortobágyi T, Granacher L. Dose-response relationships of resistance training in healthy old adults: A systematic review and meta-analysis. Am J Sports Med. 2015;45(12):1-28.

6. Guerra-Filho JL, Aniceto RR, Neto GR, et al. Validity of different prediction equations of the maximum load in mixed martial arts 
athletes. Motri. 2014;10(4):47-55.

7. Pereira FB. A influência da sarcopenia na densidade mineral óssea de homens idosos. 2014. 104f. Tese (Doutorado em Ciências da Saúde pelo Programa de Pós-Graduação em Ciências da Saúde da Universidade de Brasília). Universidade de Brasília, Brasília, 2014.

8. Lemos TV, Pereira LM. Efeitos da plataforma vibratória no sistema muscoloesquelético. Rev Movimenta. 2012;5(3):257-263.

9. Stania M, Chmielewska D, Kwana K, et al. Bioelectrical activity of the pelvic floor muscles during synchronous wholebody vibration - a randomized controlled study. BMC Urol. 2015;15(1):107-117.

10. Chiyuan MA, Liun A, Sun M, et al. Effect of whole-body vibration on reduction of bone loss and fall prevention in postmenopausal women: a meta-analysis and systematic review. J Orthop Surg Res. 2016;11(1):11-24.

11. Robinson CC, Barreto RP, Sbruzzi G, et al. The effects of whole body vibration in patients with type 2 diabetes: a systematic review and meta-analysis of randomized controlled trials. Braz J Phys Ther. 2016;20(1):4-12.

12. Mitchell UH, Hilton SC, Hunsaker E, et al. Decreased symptoms without augmented skin blood flow in subjects with RLS/WED after vibration treatment. J Clin Sleep Med. 2016;12(7):947-952.

13. Farzinmehr A, Moezy A, Koohpayehzadeh J, et al. Comparative study of whole body vibration training and pelvic floor muscle training on women's stress urinary incontinence: three- month follow- up. J Fam Plann Reprod Health Care. 2015;9(4):147154.

14. Tupimai T, Peugsuwan P, Prasertnoo J, et al. Effect of combining passive muscle stretching and whole body vibration on spasticity and physical performance of children and adolescents with cerebral palsy. J Phys Ther Sci. 2016;28(1):7-13.

15. Zhang L, Weng C, Liu M, et al. Effect of whole-body vibration exercise on mobility, balance ability and general health status in frail elderly patients: a pilot randomized controlled trial. Clin Rehabil. 2014;28(1):59-68.

16. Rogan S, De Bruin ED, Radlinger L, et al. Effects of whole-body vibration on proxies of muscle strength in old adults: a systematic review and meta-analysis on the role of physical capacity level. Eur Rev Aging Phys Act. 2015;12(1):12-38.

17. Benedetti TR, Bertoldo APC, Rodriguez-Añez CR, et al. Reprodutibilidade e validade do Questionário Internacional de Atividade Física (IPAQ) em homens idosos. Rev Bras Med Esporte. 2007;13(1):11-16.

18. Álvarez-barbosa F, Poso-cruz B, Alfonso-rosa RM, et al. Effects of supervised whole body vibration exercise on fall risk factors, functional dependence and health-related quality of life in nursing home residents aged 80+. Maturitas. 2014;79(4):456-463.

19. Sitjá-rabert M, Martínez-zapata MJ, Vanmeerhaeghe FA, et al. Effects of a whole body vibration (WBV) exercise intervention for institutionalized older people: a randomized, multicenter, parallel, clinical trial. J Am Med Dir Assoc. 2015;16(2):125-131.

20. Zaki ME. Effects of whole body vibration and resistance training on bone mineral density and anthropometry in obese postmenopausal women. J Osteoporos. 2014;2014(1):1-6.

21. Neto JP, Brito CAF. Mobilidade funcional em função da força muscular em mulheres idosas fisicamente ativas. Rev Bras Med Esporte. 2015:21(5):369-371.

22. Da Silva GM, Gass R, Rocha GG, et al. Análise comparativa da força muscular respiratória e da força muscular de membro inferior dominante entre jogadores de futebol profissional e amador. Biomotriz. 2013;6(2):30-44.

23. Avelar A, Ribeiro AS, Trindade MCC, et al. Efeito de 16 sem- anas de treinamento com pesos sobre a força muscular de mulheres não treinadas. Rev Educ Fís. 2013;24(4):649-658.

24. Almeida MHM, Litvoc J, Perez MP. Dificuldades para atividades básicas e instrumentais de vida diária, referidas por usuários de um centro de saúde escola do município de São Paulo. Rev Bras Geriatr Gerontol. 2012;15(2):187-200.

25. Barbosa BR, Almeida JM, Barbosa MR, et al. Avaliação da capacidade funcional dos idosos e fatores associados à incapacidade. Ciênc Saúde Coletiva. 2014;19(8):3316-3325.

26. ChungSin Shim RN, YunBok Lee RN, DongGeon Lee BS, et al. Effect of whole body vibration exercise in the horizontal direction on balance and fear of falling in elderly people: a pilot study. J Phys Ther Sc. 2014;26(7):1083-1086.

27. Cabello AG, González-Agüero, Ara I, et al. Effects of a shortterm whole body vibration intervention on lean mass in elderly people. Nutr Hosp. 2013;28(4):1255-1258.

28. Ferreira FR, Angeli G, Confessor, YQ, et al. Efeito agudo dos extensores do joelho unilateral na cadeira extensora com e sem estímulos na plataforma vibratória. Rev Bras Med Esporte. 2013;19(5):336-338.

29. Amaral JF, Alvim FC, Castro EA, et al. Influence of aging on isometric muscle strength, fat-free mass and electromyographic signal power of the upper and lower limbs in women. Braz $\mathrm{J}$ Phys Ther. 2014;18(2):183-190.

30. Weber FS. Ciências da Saúde pelo Programa de Pós Graduação em Ciências da Saúde da Universidade de Brasília. 2012. 105f. Pós Graduação em Ciências do Movimento Humano. Universidade Federal do Rio Grande do Sul, Porto Alegre, 2012.

31. Urso RP, Okuno NM, Batista MAB, et al. Treinamento de força com e sem o uso da plataforma vibratória é capaz de modular a variabilidade da frequência cardíaca em repouso? Rev Motriz Ed Fís. 2012;18(3):526-532.

32. Hallal CZ, Marques NR, Spinoso DH, et al. Efeito do treinamento com haste vibratória na biomecânica da marcha com dupla-tarefa em idosas. Rev Bras Med Esporte. 2014;20(6):465469.

33. Bush JA, Blog GL, Kang J, et al. Effects of quadriceps strength after static and dynamic whole-body vibration exercise. J Strength Cond Res. 2015;29(5):1367-1377.

34. Tseng SY, Lai CL, Chang KL, et al. Influence of whole-body vibration training without visual feedback on balance and lower-extremity muscle strength of the elderly: a randomized controlled trial. Am J Med. 2016;95(5):1-6.

35. Yua C, Seob SB, Kangb SR, et al. Effect of vibration on muscle strength imbalance in lower extremity using multicontrol whole body vibration platform. Biomed Mater Eng. 2015;26:673-683.

36. Yang F, Rei GA, Dillon L, et al. Controlled whole-body vibration training reduces risk of falls among community-dwelling older adults. J Biomech. 2016;48(12):3206-3212.

37. Duguette SA, Giulliano AM, Starmer DJ. Whole body vibration and cerebral palsy: a systematic review. J Can Chiropr Assoc. 2015;59(3):245-2015.

38. Yabumoto T, Shin S, Watanabe T, et al. Whole-body vibration training improves the walking ability of a moderately impaired child with cerebral palsy: a case study. J Phys Ther Sci. 2015;27(9):3023-2025.

39. Giombini A, Menotti F, Laudani L, et al. Effect of whole body vibration frequency on neuromuscular activity in ACL-deficient and healthy males. Biol Sport. 2015;32(3):243-247.

40. Park SY, Filho WM, Kwon OS. Effects of whole body vibration training on body composition, skeletal muscle strength, and cardiovascular health. J Exerc Rehabil. 2015;11(6):289-295. 